\title{
Evolución geoquímica de la Formación Eagle Ford y su rol como roca generadora de gas y aceite en Coahuila, NE México
}

\section{Geochemical evolution of the Eagle Ford Formation and its role as source rocks for gas and oil in Coahuila, NE Mexico}

\author{
Alberto Herrera Palomo ${ }^{1, *}$, Luis Martínez ${ }^{2}$
}

${ }^{1}$ Facultad de Ingeniería, Universidad Nacional Autónoma de México, Ciudad Universitaria, 04510, Coyoacán, CDMX, México.

${ }^{2}$ EOST, Université de Strasbourg, 5 Rue René Descartes, 67000, Strasbourg, Francia.

*Autor para correspondencia: (A. Herrera Palomo)a.herrera@unam.mx

\section{RESUMEN}

Con el objetivo de determinar la calidad de la Formación Eagle Ford, en la plataforma Burro - Picachos, como roca generadora de hidrocarburos y su implicación en el contexto de un yacimiento de tipo no convencional (shale gas), se analizaron con la adaptación del método Dykstra - Parson para la permeabilidad, los valores promedios ponderados al espesor de COT, Tmax, S2/S3 y PP (1.42 a 6.1) de cuatro pozos ubicados en la porción sureste de la línea fronteriza del estado de Coahuila, en el intervalo de $1410 \mathrm{~m}$ a $2460 \mathrm{~m}$ de profundidad. Gracias al contenido de COT (1.05 a 2.44\%), presente en el área de estudio, la Formación Eagle Ford se puede catalogar como generadora de hidrocarburos, con una calidad regular a excelente. Los datos de Tmax $\left(443^{\circ} \mathrm{C} \mathrm{a} 484^{\circ} \mathrm{C}\right)$, ubican la roca generadora en una fase de madurez térmica temprana a tardía, a excepción del pozo Habano-1 que llegó a la sobre madurez. El potencial productor $(\mathrm{PP})$ de la zona se encuentra entre débil a medio y el cálculo de tipo de hidrocarburo generado (S2/S3) indica la presencia de aceite y gas en la formación. Esto podría limitar la existencia de aceite a profundidades máximas de $1820 \mathrm{~m}$ en la porción norte del área estudiada. Si se observan el índice de hidrógeno $(\mathrm{IH})$ vs. índice de oxígeno (IO), su evolución nos indica que el kerógeno predominante es una mezcla formada principalmente del tipo III y II los cuales se pueden interpretar como los precursores del gas y aceite encontrado. De los cuatro pozos exploratorios analizados, sólo uno presenta características geoquímicas con valores de corte similares a los propuestos en el desarrollo de shale gas en la Cuenca de Barnett.

Palabras clave: Eagle Ford, kerógeno, shale gas, Carbono Orgánico Total, hidrocarburos.

\section{ABSTRACT}

To determine the quality of the Eagle Ford Formation, on the Burro - Picachos platform, as a hydrocarbon generating rock and its implication in the context of an unconventional type of deposit (shale gas), they were analyzed with the adaptation of the method Dykstra - Parson for permeability, the average values weighted to the thickness of COT, Tmax, S2 / S3 and PP 1.42 to 6.1) of four wells located in the southeast portion of the border line of the state of Coahuila, in the range of $1410 \mathrm{~m} 2460 \mathrm{~m}$ deep. Thanks to the COT content (1.05 to 2.44\%), present in the study area, the Eagle Ford Formation can be classified as a hydrocarbon generator, with a regular to excellent quality. The Tmax data (443 ${ }^{\circ} C$ at $484{ }^{\circ} \mathrm{C}$ ), locate the generating rock in a phase of early to late thermal maturity, with the exception of the Habano-1 well that reached over maturity. The potential producer (PP) of the area is between weak to medium and the calculation of the type of hydrocarbon generated (S2 / S3) indicates the presence of oil and gas in the formation. This could limit the existence of oil at maximum depths of $1820 \mathrm{~m}$ in the northern portion of the studied area, if the hydrogen index (IH) is observed. Oxygen index (IO), its evolution indicates that the predominant is a mixture formed mainly of type III and II which can be interpreted as the precursors of the gas and oil found of four exploratory wells analyzed, only one presents geochemical characteristics with cutoff values similar to those proposed in shale gas development in the Barnett Basin.

Keywerds: Eagle Ford, kerogen, shale gas, Total Organic Carbon, hydrocarbons. 


\section{Introducción}

La Agencia de Información de Energía (EIA por su siglas en inglés) ubica a México entre los primeros diez países con reservas técnicamente recuperables en yacimientos tipo shale gas y shale oil, en el sexto sitio en reservas de gas con 545 tcf y en el octavo en reservas de aceite con $13 \mathrm{mmmb}$ (DOE/EIA-0383, 2016). La Comisión Nacional de Hidrocarburos $(\mathrm{CNH})$ ubica estos recursos en 6 zonas a lo largo de porción nororiental del país; En la Figura 1 se muestran las zonas de: 1) Chihuahua. 2) Sabinas. 3) Burro - Picachos.

4) Burgos. 5) Tampico - Misantla y 6) Veracruz (CNH, 2016).

Meneses S. J. (2015) en su trabajo doctoral, apoyado en el contexto regional proporcionado por Humphrey (1958), cita que durante el Cenomaniano -Turoniano tardío, una transgresión global produjo un ciclo transgresivo que permitió la comunicación entre el Golfo de México y el mar interior occidental. Los sedimentos terrígenos además calcáreos fueron depositados en el este y noreste de México en facies someras, intermedias y profundas, donde se han descrito con los nombres de Formación Indidura, Formación Agua Nueva y Formación Eagle Ford; ésta última cubre las cuencas de Sabinas y la plataforma Burro-Picachos.En México, la Formación Eagle Ford, en particular en la plataforma Burro - Picachos, ha sido considerada como una formación generadora de hidrocarburos tipo shale gas, motivo por el cual, Petróleos Mexicanos (PEMEX) a través de PEMEX Exploración Producción (PEP) recientemente ha realizado una serie de pozos exploratorios a lo largo de la frontera de Coahuila con Texas (Tellez, 2011), obteniendo como resultado 2 pozos productores de gas seco (Emergente-1, Percutor-1), 2 productores de gas seco y condensado (Habano -1, Chucla-1); 2 productores no comerciales de gas y condensado (Montañes-1; Gamma-1) y 1 pozo improductivo seco (Nómada-1) (CNH 2016).El área estudiada se delimitó en función de la información recabada de 4 pozos exploratorios: Montañes-1, Gamma-1, Habano-1 y Emergente-1, cuya ubicación se muestra en la Figura 2.

La columna estratigráfica del Cretácico en los pozos mencionados está constituida por las

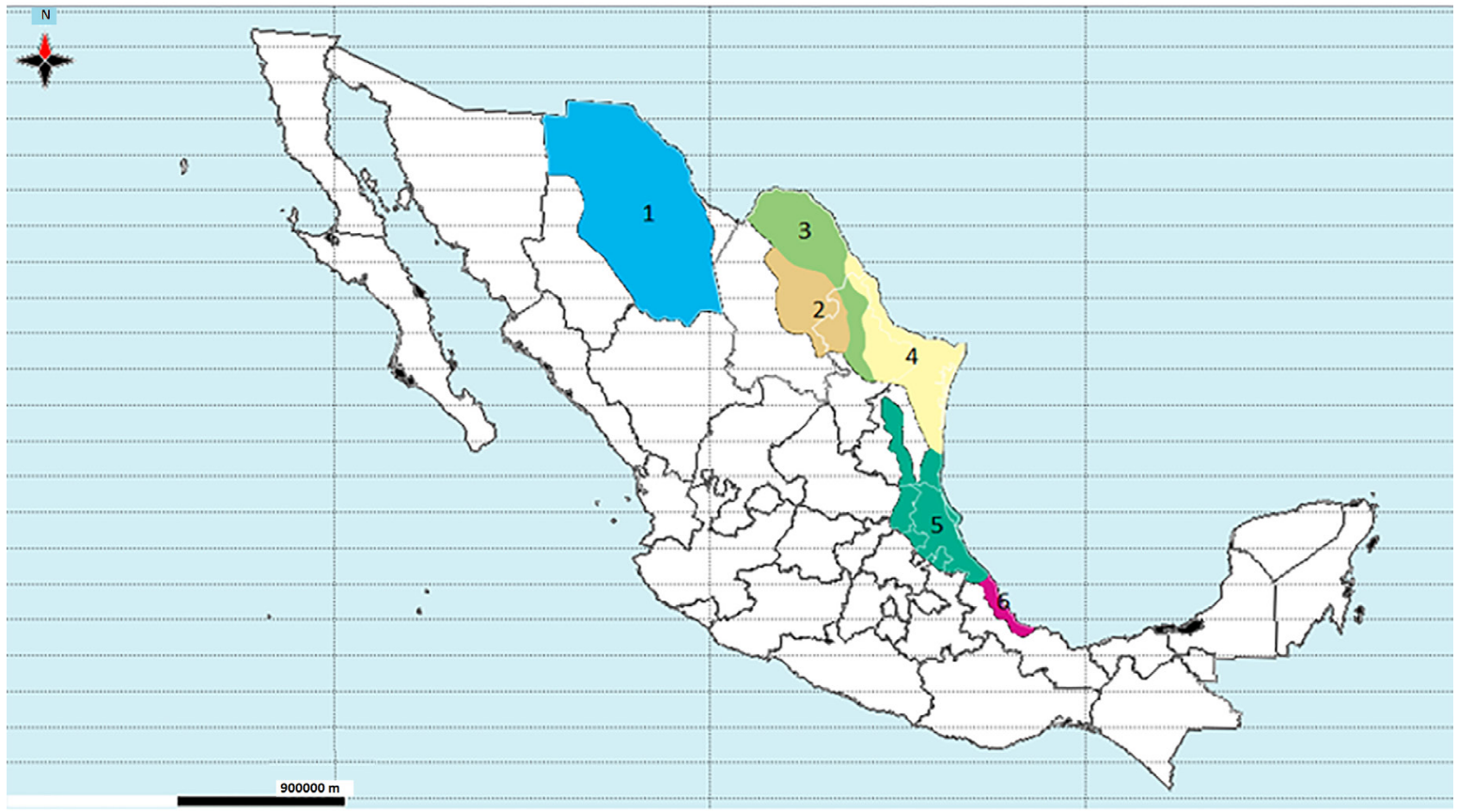


formaciones del Cenomaniano inferior al Maastrichtiano, las cuales son: Buda, Eagle Ford, Austin, San Miguel, Olmos y Escondido (Figura 3). Dichas formaciones han sido descritas por diversos autores (Padilla y Sánchéz (1982), Barcelo Duarte (1983) y Meneses (2015), entre otros).

Este trabajo tiene como objetivo determinar con el grado de madurez térmica actual de la materia orgánica presente en la Formación Eagle Ford en cada uno de los 4 pozos, su implicación en la generación de aceite - gas, y su consideración para la realización futura de un proyecto tipo shale en esta región.

\section{Metodología}

Para definir si la Formación. Eagle Ford se puede clasificar como una roca generadora de hidrocarburos, se empleo un análisis Rock-Eval para determinar el contenido de Carbono Orgánico Total presente (COT \% peso), la Temperatura máxima Rock-Eval $\left(\operatorname{Tmax}{ }^{\circ} \mathrm{C}\right)$, el tipo de hidrocarburos $(\mathrm{S} 2 / \mathrm{S} 3)$ y el Potencial Productor (PP $=\mathrm{S} 1+\mathrm{S} 2)$. Aunque para determinar si se usa en los cálculos un promedio simple, ponderado al espesor, geométrico o armónico, se utilizó el Coeficiente Dykstra - Parson, esta es una herramienta estadística para medir la heterogeneidad de la permeabilidad estática en un bloque de datos, pero puede ser extrapolada a otras propiedades como lo indica Celio Maschio (2003). La tabla 1 indica los valores del coeficiente de heterogeneidad (V) de Dykstra - Parson.

Los pasos principales que se suguieren para calcular el índice de heterogeneidad (Dykstra Parson) son los siguientes:(i) Organizar los datos en orden decreciente, (ii) calcular para cada valor el porcentaje de valores de probabilidad acumulada expresada en \%, (iii) graficar los datos del paso anterior en papel de probabilidad logarítmica. Donde se traza el valor en el eje de las ordenadas mientras la probabilidad acumulada en el eje de las abscisas. (iv) ajustar los datos a una línea recta y obtener los valores de $\mathrm{P} 50 \%$ y $\mathrm{P} 84.1 \%$, (v) calcular el índice de heterogeneidad de acuerdo a $\mathrm{V}=$ (P 50 - P 84.1) / P 50.

Gracias a los valores promedios ponderados del espesor de COT, y los parámetros Tmax, S2/ S3 y PP se pudo construir la Tabla 2, donde se

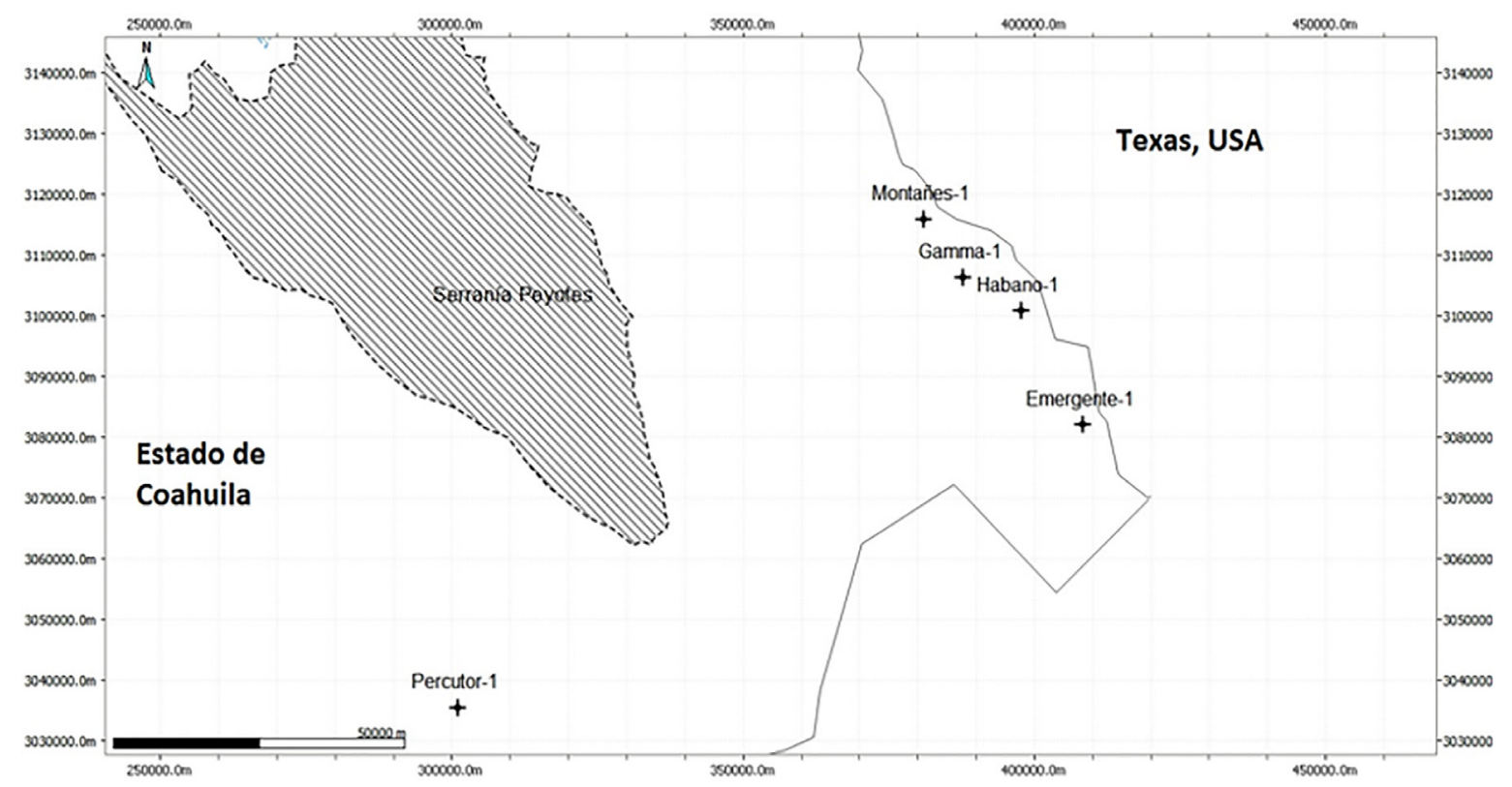


muestran las características geoquímicas del área de estudio.Para determinar la factibilidad de realizar un proyecto de un yacimiento tipo shale en esta región, se analizó, además de la cantidad de COT y Tmax, el Valor de reflectancia calculada de la vitrinita $(\mathrm{VRc} \%$ ) y el Índice de transformación de kerógeno a gas y aceite $(\operatorname{Tr} \%)$. Se decidió realizar el cálculo del \%VRc, pero al no contar con acceso a los datos en la región, entonces se utilizaron las ecuaciones propuestas por Jarvie et al, (2005) para los cálculos de los parámetros citados.

Con respecto al valor de reflectancia calculada de la vitrinita, se sabe que esto no es lo más recomendable, ya que se tiene mayor certeza al utilizar valores de VRo medidos en macerales de las muestras, por lo que se debe de tomar con prudencia la entrada del kerógeno en la ventana del aceite y el gas y su evaluación en el prospecto tipo shale gas de los cuatro pozos.

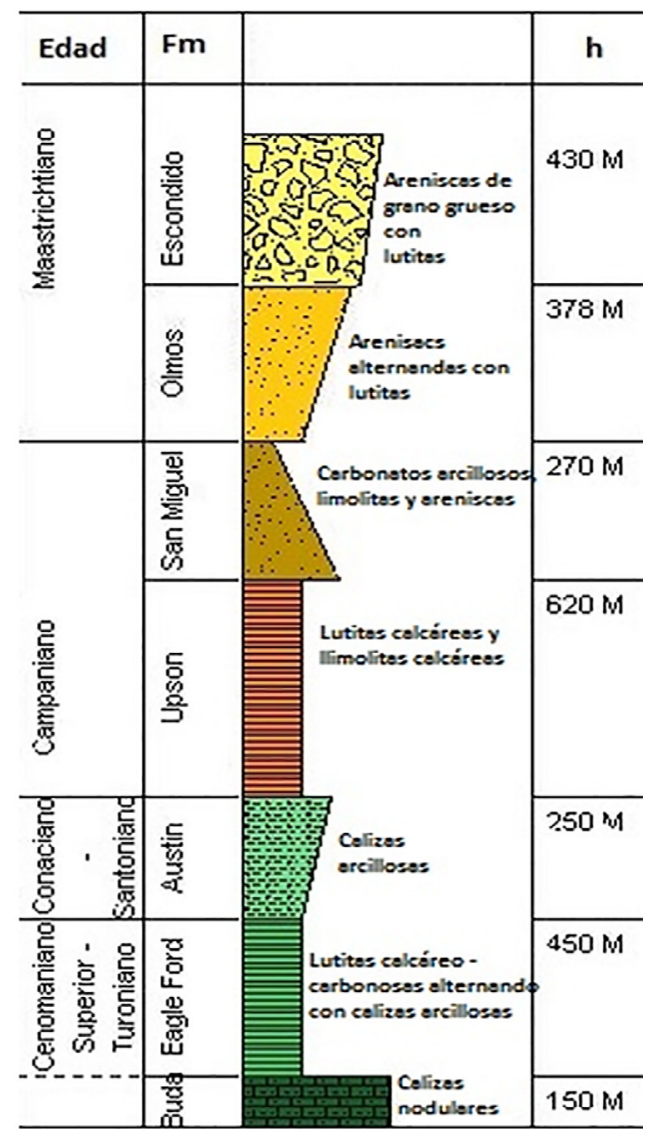

Figura 3 Columna estratigráfica en la plataforma Burro-Picachos (Elaborada a partir de informes técnicos de PEMEX;Tellez,2011).
Tabla 1 Coeficiente de heterogeneidad calculado por el método de Dykstra - Parson y uso de valores promedio.

\begin{tabular}{|c|}
\hline \\
\hline $0.0-0.25$ ligeramente heterogéneo (promedio aritmético). \\
$0.25-0.50$ heterogéneo medio (promedio ponderado). \\
$0.50-0.75$ muy heterogéneo (promedio geométrico). \\
$0.75-1.00$ completamente heterogéneo (promedio armónico).
\end{tabular}

- Valor de la reflectancia (VRo) de la vitrinita:

Cal. $\%$ VRc $($ de Tmax $)=0.0180 \times \operatorname{Tmax}-7.16[\%]$

- COT original

COT original calculado $=$ COT actual $/ 0.64$ [wt. \% ]. (2)

- El potencial de generación original se puede calcular utilizando el valor promedio de carbono contenido en los hidrocarburos $(83 \%)$ e incrementando el potencial generador $\mathrm{S} 2$.

COT transformado $=$ COT original -

$$
\text { COT presente [wt. \% ] }
$$

$\mathrm{S} 2$ original $=\mathrm{COT}$ original $/ 0.083+\mathrm{S} 2$

$$
\text { presente [mg hc's /g rock] }
$$

- Valor original del índice de hidrógeno (IH):

$\mathrm{IH}$ original $=\mathrm{S} 2$ original $/ \mathrm{COT}$ original $\mathrm{x} 100$

$$
\text { [mg hc's/g COT] }
$$

- Relación de transformación del kerógeno (Tr).

$\operatorname{Tr}$ (índice de Transformación $)=(\mathrm{IH}$ original $-\mathrm{IH}$ presente) / IH original [\%]

El contenido de COT (\% peso) es fundamental para determinar la presencia de una roca generadora de hidrocarburos, por lo que es necesario conocer su distribución espacial. En esta investigación 
se emplearon los programas computacionales SURFER y MOVE, ya que ambos realizan la interpolación y extrapolación por medio de la geoestadística (kriging). El mapa de distribución de COT que se obtuvo se muestra en la Figura 4.

\section{Resultados y análisis}

\subsection{DISTRIBUGIÓN ESPAGIAL DEL GONTENIDO DE COT}

Como se observa en la Figura 4, el contenido promedio de COT se distribuye de manera más uniforme en una dirección NE - SW, con valores promedio de $1 \%$ a $2 \%$ en los pozos Gamma-1, Montañés- 1 y Emergente-1, mientras el pozo Habano-1 presentó más del 2\% de COT. De las muestras estudiadas, se observa que el pozo Habano -1 presenta una mayor riqueza orgánica respecto a los pozos aledaños al NW (Montañes-1 y Gamma-1), y al SE (Emergente-1), por lo cual se considera que esta zona posee un contenido orgánico indicativo de una roca generadora de buena calidad.

\section{2. ÍNDIGE DE HETEROGENEIDAD (V)}

La distribución del contenido de COT (Figura 5) presentó un índice de heterogeneidad ligero en el pozo Habano - 1 ( $\mathrm{V}=0.25)$, mientras que en los pozos Emergente -1, Montañés -1 y Gamma

\begin{tabular}{|l|c|c|c|c|}
\hline \multicolumn{1}{|c|}{ Tabla 2 Características geoquímicas en el área de estudio. } \\
\hline Pozo & $\begin{array}{c}\text { COT } \\
\text { (peso \%) }\end{array}$ & $\begin{array}{c}\text { Tmax } \\
\left({ }^{\circ} \mathrm{C}\right)\end{array}$ & S2/S3 & PP \\
\hline Emergente -1 & 1.12 & 443 & 3.2 & 1.69 \\
\hline Gamma-1 & 1.70 & 446 & 2.65 & 6.1 \\
\hline Habano-1 & 2.44 & 484 & 2.3 & 1.42 \\
\hline Montañes-1 & 1.05 & 448 & 4.0 & 1.86 \\
\hline
\end{tabular}

-1, la distribución de COT presentó un grado de heterogeneidad medio $(\mathrm{V}=0.32$ a 0.42$)$. Por su parte, la variación en los valores de Tmax presentó un grado ligero de heterogeneidad en los cuatro pozos analizados con valores de 0.007 a 0.06 .

El índice de heterogeneidad para los parámetros geoquímico de $\mathrm{S} 2 / \mathrm{S} 3(\mathrm{~V}=0.18$ a 0.76$)$ y $\mathrm{PP}$ $(\mathrm{V}=0.24$ a 0.69$)$ le confiere a la zona un grado de heterogeneidad de ligero a muy heterogéneo, siendo el pozo Habano -1 el que mayor índice de heterogeneidad presentó para ambos parámetros. De acuerdo con el método basado en el Coeficiente de Dykstra - Parson, los cuatro pozos presentaron un valor $\mathrm{V}$ de ligero a medianamente heterogéneo para los parámetros COT y Tmax, mientras que para los parámetros S2/S3 y $\mathrm{PP}$, tres pozos presentaron $\mathrm{V}$ de ligero a medianamente heterogéneo, y solo el pozo Habano-1 presentó V como muy heterogéneo, por lo que el uso del promedio ponderado al espesor para su interpretación es considerado el más adecuado.

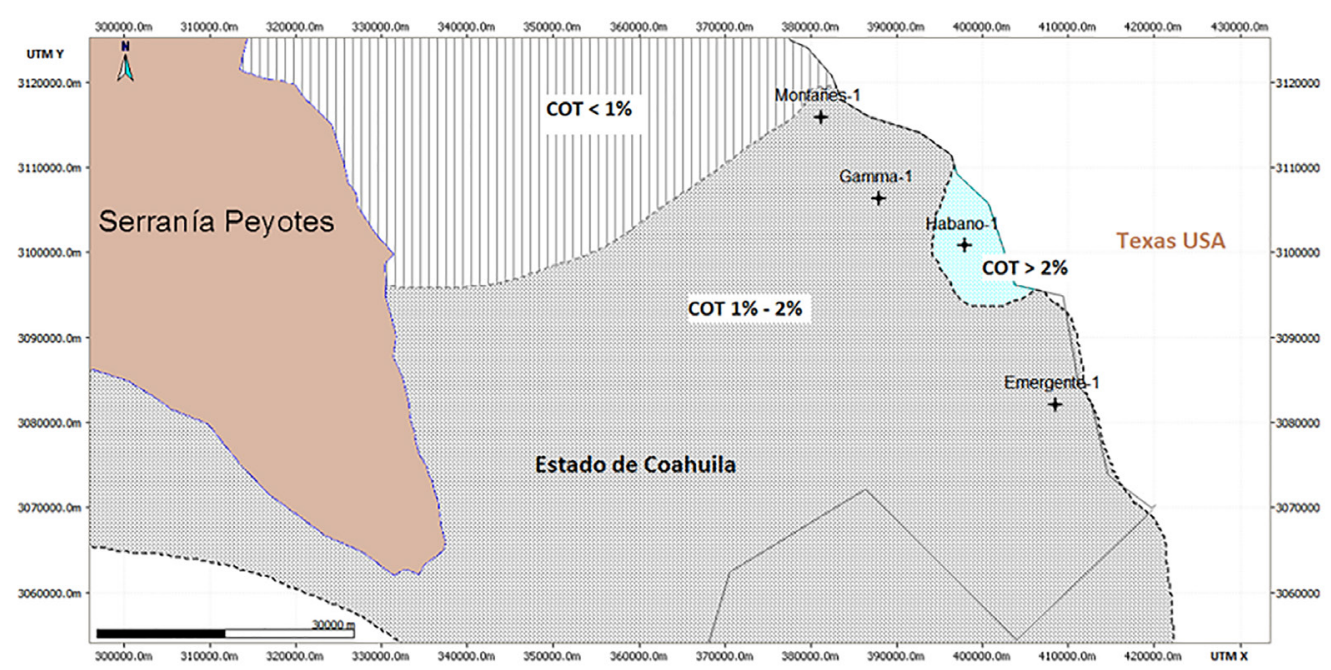

Figura 4 Mapa de distribución del COT (peso \%) de la Fm. Eagle Ford. Con la ubicación de los pozos estudiados. 


\subsection{GONTENIDO DE GOT (\% PESO) PRESENTE}

De la Figura 6, en general, se interpretó el contenido de COT (\% peso) en cada uno de los pozos, aunque se observa una mayor concentración conforme se incrementa la profundidad, de tal manera que para el pozo Montañés -1 este incremento se da a partir de los $1460 \mathrm{~m}$ y hasta los 1560 $\mathrm{m}$ de profundidad, con valores de 1.0 a 2 COT ( $\%$ peso). Mientras que en el Pozo Habano-1 la concentración de COT es más homogénea en profundidades de $1960 \mathrm{~m}$ a $2060 \mathrm{~m}$ con contenido de 1.0 a 2.6 de COT (\% peso). Por su parte, el pozo Emergente - 1 presentó una mejor concentración de COT entre los $2410 \mathrm{~m}$ a 2,480 m con valores de $1.0(\%$ peso) a $2.0(\%$ peso).Con base a la información anterior, se confirma que, aunque la Formación Eagle Ford presenta variación de espesor y profundización en esta área, posee un espesor de $100 \mathrm{~m}$ que se puede catalogar como generadora de hidrocarburos, con una calidad de buena a excelente.

En la Figura 6, de manera adicional, se incluyeron datos del pozo Percutor-1; este pozo se encuentra ubicado al occidente del área de análisis, cuya ubicación se muestra en la Figura 2.
El contenido de COT está relacionado al paleoambiente y paleogeografía que representa la Formación Eagle Ford, la cual es marina somera a marina profunda, en conjunto con el levantamiento y plegamiento diferencial de esa zona del país, lo que explica esas diferencias en riqueza orgánica. El COT está aparentemente relacionado con la naturaleza de las lutitas y con sus características petrofísicas analizadas además se puede considerar que la formación estudiada es un shale que tiene un comportamiento, a partir de esta información, de tipo sistema petrolero no convencional.

\subsection{TEMPERATURA MÁXIMA [TMAX $\left.\left({ }^{\circ} \mathrm{C}\right)\right]$}

La Figura 8 muestra que, para los pozos Montañés-1 y Gamma-1, la Tmax de Rock-Eval reportada en los estudios geoquímicos inició con valores cercanos a los $433^{\circ} \mathrm{C}$, lo que los situó en una etapa de madurez temprana.

Esta temperatura mostró un incremento conforme se profundiza la Formación Eagle Ford, pero después de los $452^{\circ} \mathrm{C}$ presentó una disminución en los valores de temperatura sin salir de la etapa de madurez que se podría asociar a una variación

Índice de heterogeneidad

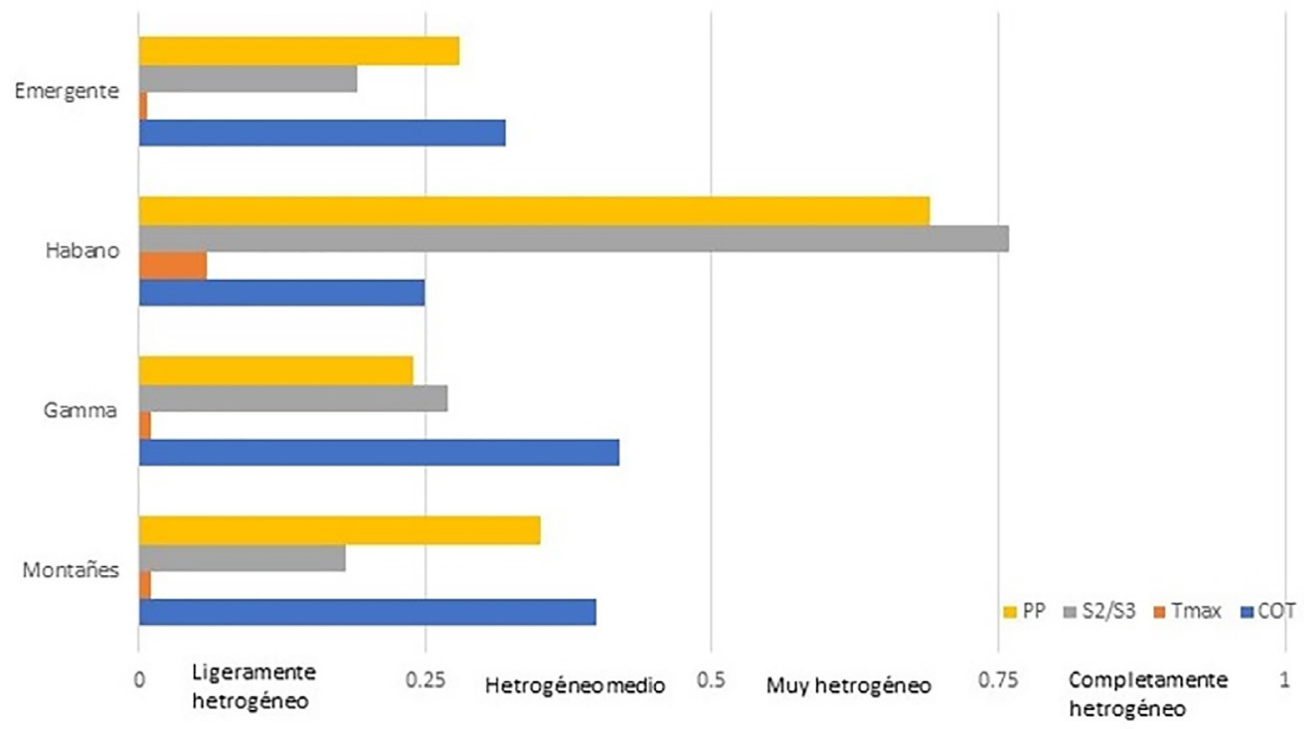

Figura 5 Índice de heterogeneidad calculado para cada uno de los pozos analizados. En el pozo Gamma-1 se dio una mejor concentración de COT entre los $1710 \mathrm{~m}$ a $1810 \mathrm{~m}$ de profundidad, aunque llegaron a presentar valores de 5.3 (\% peso) La mejor concentración se dio en los valores de 1.5 (\% peso) a 2.4 (\% peso). 
de composición orgánica en la sedimentación por un levantamiento tectónico regional diferenciado del área. En el pozo Emergente -1, los valores de Tmax se agruparon esencialmente entre las temperaturas de $430^{\circ} \mathrm{C}$ a $450^{\circ} \mathrm{C}$, alcanzando la etapa de madurez media; sin embargo, presentaron como característica particular un descenso de la temperatura conforme se incrementa la profundidad, esto se podría interpretar como el resultado de un cambio en el gradiente geotérmico. Por su parte, el pozo Habano-1 presentó una mayor distribución de los valores de COT, los cuales abarcan valores de $432^{\circ} \mathrm{C}$ hasta $610^{\circ} \mathrm{C}$, pero el $70 \%$ de ellos se concentraron entre $\operatorname{los} 438^{\circ} \mathrm{C}$ a $449^{\circ} \mathrm{C}$, lo que nos indicó que este pozo entró a la etapa de madurez para la generación de aceite y probablemente de gas.

Los valores de Tmax de los pozos analizados, indicaron de manera general que la Formación Eagle Ford alcanzó la etapa de madurez térmica necesaria para la explotación de la generación de aceite y gas.

\subsection{IH VERSUS IO}

En el pseudodiagrama de Van Krevelen, los valores de Índice de Hidrógeno ( $\mathrm{IH}$ ) contra Índice de
Oxigeno (IO) (Figura 7),muestran que el tipo de kerógeno predominante en el área es del tipo III, aunque también se tiene presente kerógeno tipo II en los pozos Montañés -1 y Gamma-1.

$\mathrm{Al}$ analizar el Índice de Hidrógeno se observó que, en los Pozos Habano - 1 y Emergente -1, los kerógenos presentes fueron precursores de gas y condensado, mientras para que los pozos Montañés -1 y Gamma -1, además de gas y condensado, fue precursor de aceite.

\section{6. $\mathrm{S} 2 / \mathrm{S} 3$}

El Tmax de la Figura 8 y el índice S2/S3 de la figura 9 no proporcionan información sobre la madurez térmica de la materia orgánica estudiada y su potencial para producir aceite - aceite + gas gas, sobre todo en la etapa de catagénesis.

En la Figura 9 se observa que los pozos Montañés - 1 y Gamma -1 son potencialmente generadores de aceite y condensado, ya que los valores de S2 / S3 van de 2 hasta 6.5, mientras que los pozos Habano-1 y Emergente -1 son principalmente potenciales productores de condensado (S2 / S3 $=1.7$ a 4.8), por lo que se interpreta que la presencia de aceite se limita a los pozos ubicados en la

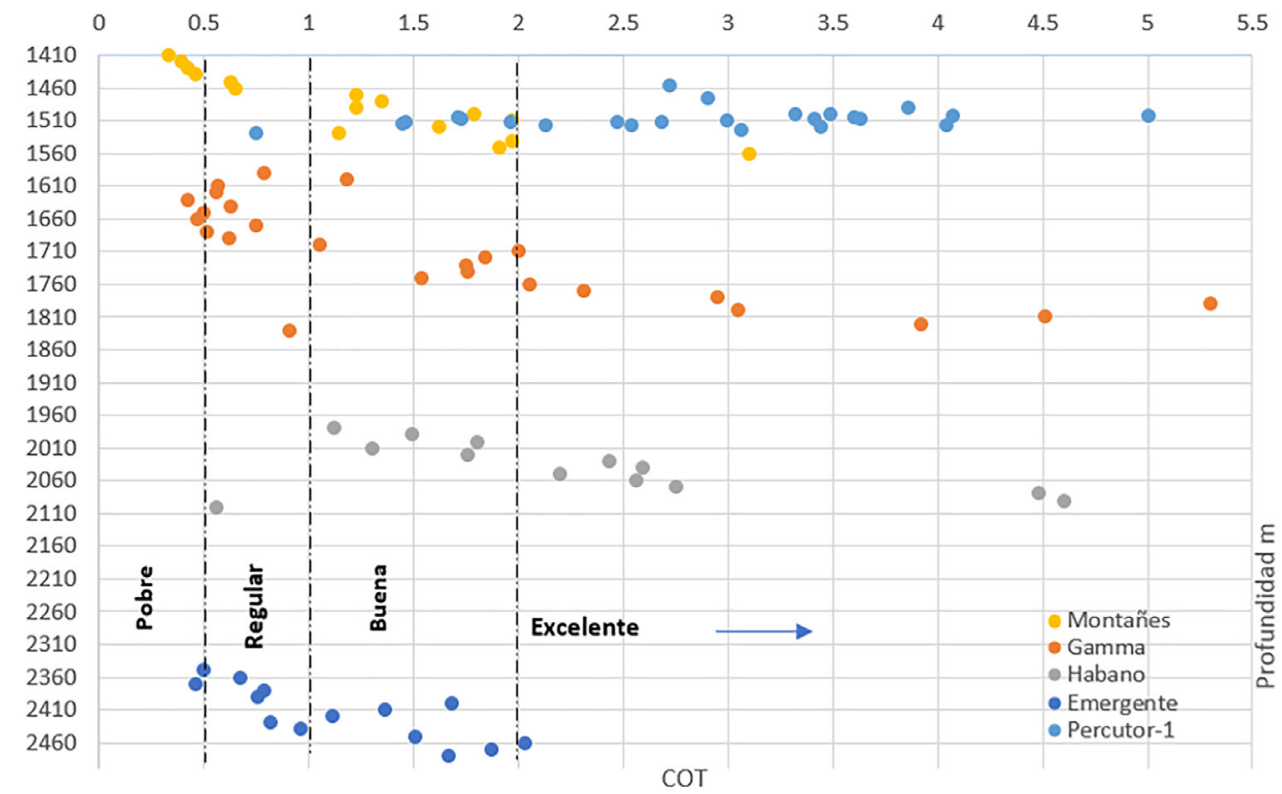

Figura 6 Clasificación de la calidad de la roca como generadora de hidrocarburos en función del contenido de COT (Contenido Orgánico Total). 
IH vs 10

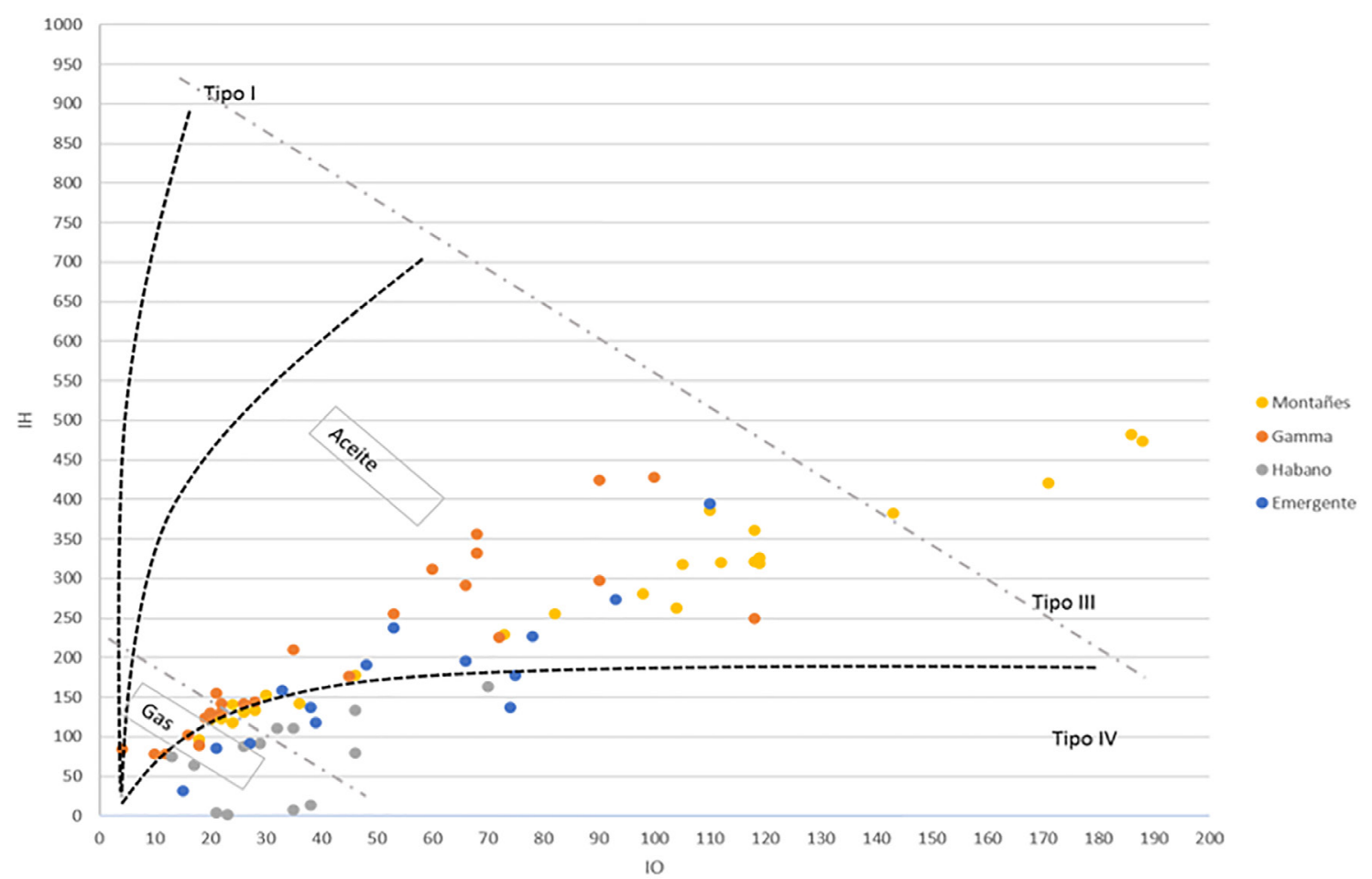

Figura 7 Pseudo diagrama de Van Krevelen que muestra el tipo de kerógeno y su evolución térmica predominante en el área de estudio.

porción norte del área estudiada, en un intervalo de profundidad de $1460 \mathrm{~m}$ a $1560 \mathrm{~m}$ para el pozo Montañes-1 y de 1560 m a 1810 m en el pozo Gamma-1. La profundidad que podría considerarse para tener gas con aceite está puntualmente localizada en el pozo Habano-1 en $2060 \mathrm{~m}$.

\subsection{VRO $(\%)$, TR $(\%)$}

Los valores calculados por medio de las ecuaciones propuestas por Jarvie et al. (2005) para los pozos analizados se muestran en la Tabla 3.

Si comparamos los resultados en esta tabla con los valores de las características geoquímicas de la Cuenca Barnett en USA (Tabla 4) que muestran

Tabla 3 Valores de VRc, Tmax, y Tr de los pozos analizados.

\begin{tabular}{|l|c|c|c|c|}
\hline Parámetro & Montañes-1 & Gamma-1 & Habano-1 & Emergente-1 \\
\hline $\operatorname{VRc}(\%)$ & 0.89 & 0.83 & 1.77 & 0.80 \\
\hline $\operatorname{Tmax}\left({ }^{\circ} \mathrm{C}\right)$ & 448 & 446 & 484 & 442 \\
\hline $\operatorname{Tr}(\%)$ & 58 & 63 & 90 & 69 \\
\hline
\end{tabular}

Tabla 4 Valores de corte de Tmax, Tr y VRo, para un play de gas shale (Jarvie 2005).

\begin{tabular}{|l|l|l|}
\hline $\operatorname{Tmax}\left({ }^{\circ} \mathrm{C}\right)$ & $\operatorname{Tr}(\%)$ & VRo $(\%)$ \\
\hline 455 & 80 & 1.0
\end{tabular}

los valores de corte propuestos por Jarvie (2007), se observa que el pozo Habano-1, a pesar de ser muy heterogéneo en su Potencial Productor (PP) y tipo de hidrocarburo (S2 / S3), los valores rebasan los valores de corte de la Guenca de Barnett, lo que puede indicar mejor rentabilidad, dado que su potencial es más afín a generar aceite (Figura 10).

\section{Discusión}

En el análisis de datos obtenido se observa la presencia de valores altos de Tmax, principalmente en el pozo Habano - 1. La limitación de la cantidad de pozos y datos analizados no permite conocer con precisión la historia térmica de cada pozo y, por lo tanto, la evolución térmica del área, esto 
$\begin{array}{lllllllllllllllllllll}420 & 430 & 440 & 450 & 460 & 470 & 480 & 490 & 500 & 510 & 520 & 530 & 540 & 550 & 560 & 570 & 580 & 590 & 600 & 610 & 620\end{array}$

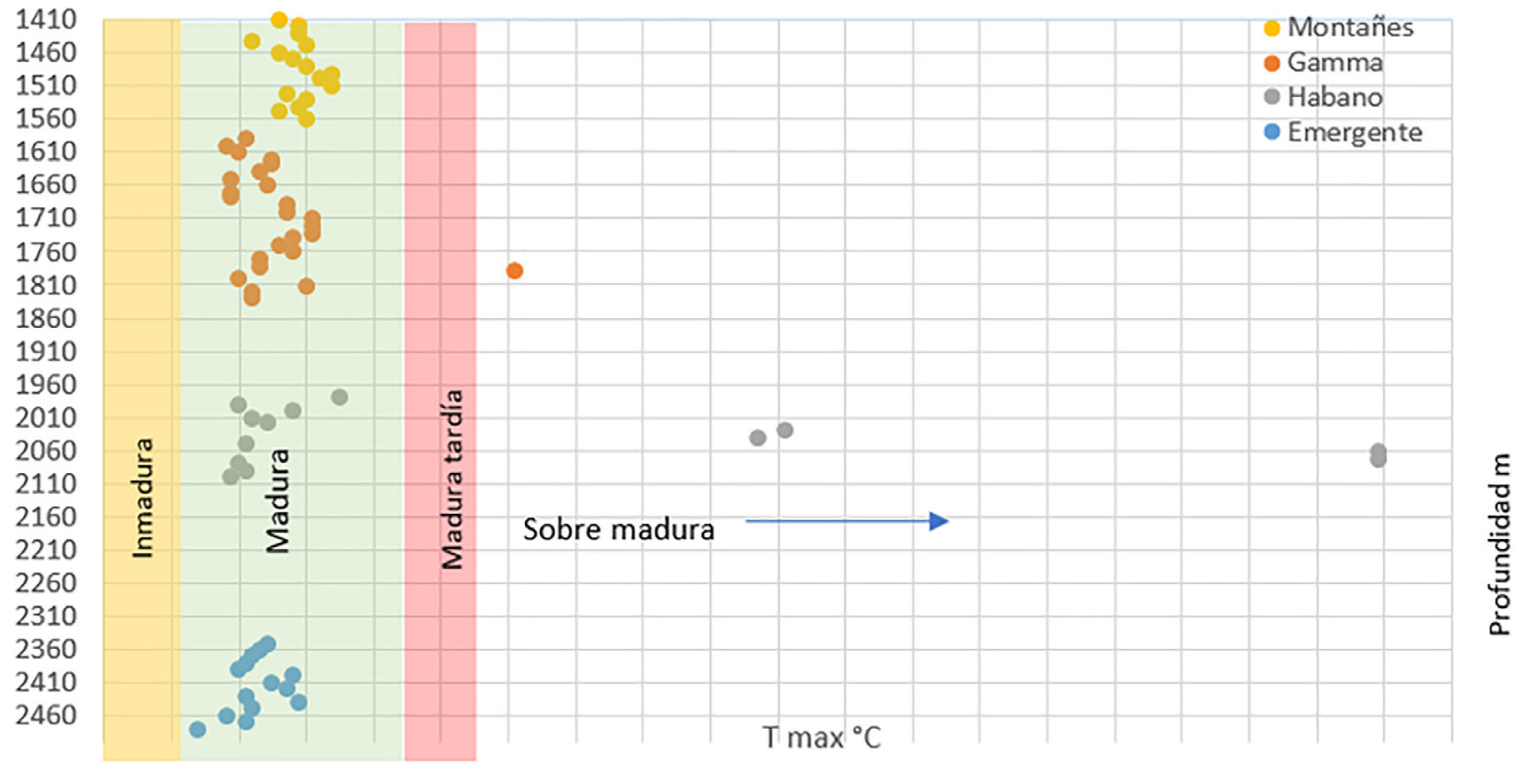

Figura 8 Variación de $\operatorname{Tmax}\left({ }^{\circ} \mathrm{C}\right)$ en función de la profundidad.

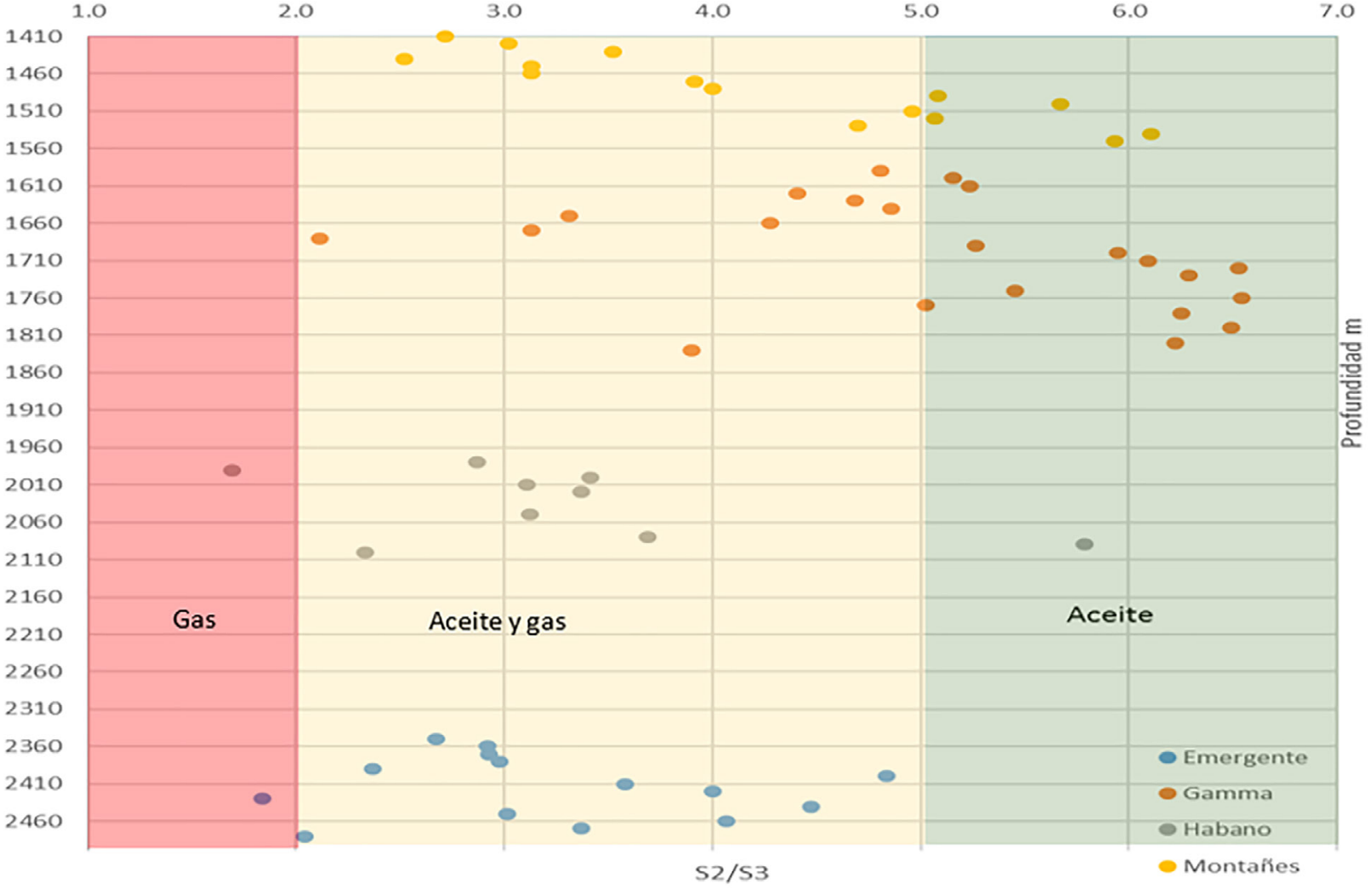

Figura 9 Valores de S2/S3 contra profundidad. 
debido a que no se contó con datos de gradiente geotérmico y de reflectancia de la vitrinita (VRo).

Pensamos que estos valores de VRc se pueden considerar como anómalos; sin embargo, de acuerdo con la evolución tectónica del área, el levantamiento regional durante el Terciario tardío, provocó la exposición de una erosión generalizada de las rocas del Mesozoico en la mayor parte de noreste de México; a excepción de la Cuenca de Burgos, que fue sometida a una subsidencia rápida durante el Terciario, lo que pudo influir en el incremento de la madurez térmica hacia el sureste del área analizada.

El análisis de los parámetros de COT, Tmax, $\mathrm{S} 1 / \mathrm{S} 2$ e IH, realizado a los 4 pozos ubicados en la plataforma de Burro - Picachos, indicó la presencia de características de roca generadora en todo el intervalo estudiado, con un mayor potencial de aceite, aceite y gas asociado y menor gas no asociado hacia la parte somera, en la parte profunda, hacia el sur de la región estudiada.

Asímismo, se encontró que los dos pozos emplazados en el norte del área analizada (Montañés - 1 y Gamma - 1), son propensos a generar aceite, mientras que los situados en la zona sur (Habano -1 y Emergente -1) son generadores principalmente de gas asociado. Estas características de mejor calidad y tipo de hidrocarburo generado son similares a las descritas en esta Formación en la porción correspondiente a los Estados Unidos de Norte América. Una parte del intervalo, Montañes-1 y Gamma-1, presenta potencial de generación de aceite, aceite y gas asociado y menor gas no asociado; mientras, Habano-1 y Emergente-1, sólo sugieren el potencial de aceite y gas asociado y gas no asociado en el mismo intervalo. De este modo, Montañes-1 y Gamma-1 son más similares a lo descrito para la Cuenca de Barnett.

\section{Conclusiones}

La Formación Eagle Ford, como lo indican sus propiedades geoquímicas de COT, IH/IO, Tmax, S2/S3 y PP, posee un contenido de carbono orgánico total de regular a excelente, proveniente de kerógeno tipo III y II. Entró a la ventana de generación de hidrocarburos en etapa de madura temprana, madura tardía y sobre madura, produciendo gas, condensado y aceite, aunque su potencial de producción es débil a medio y se le puede considerar como una formación generadora de hidrocarburos.

El Pozo Habano-1, ubicado en la Formación Eagle Ford al SE, presenta mejores valores de Tmax, VRo, y TR, por lo que se puede considerar como una zona apropiada para el desarrollo de un proyecto tipo gas shale a partir de los 1960 $m$ de profundidad.La distribución espacial del contenido de COT, en el área de estudio, presenta menor variación en dirección $\mathrm{NE}-\mathrm{SW}$, por lo que las demás propiedades geoquímicas se deben de analizar en esta misma dirección.
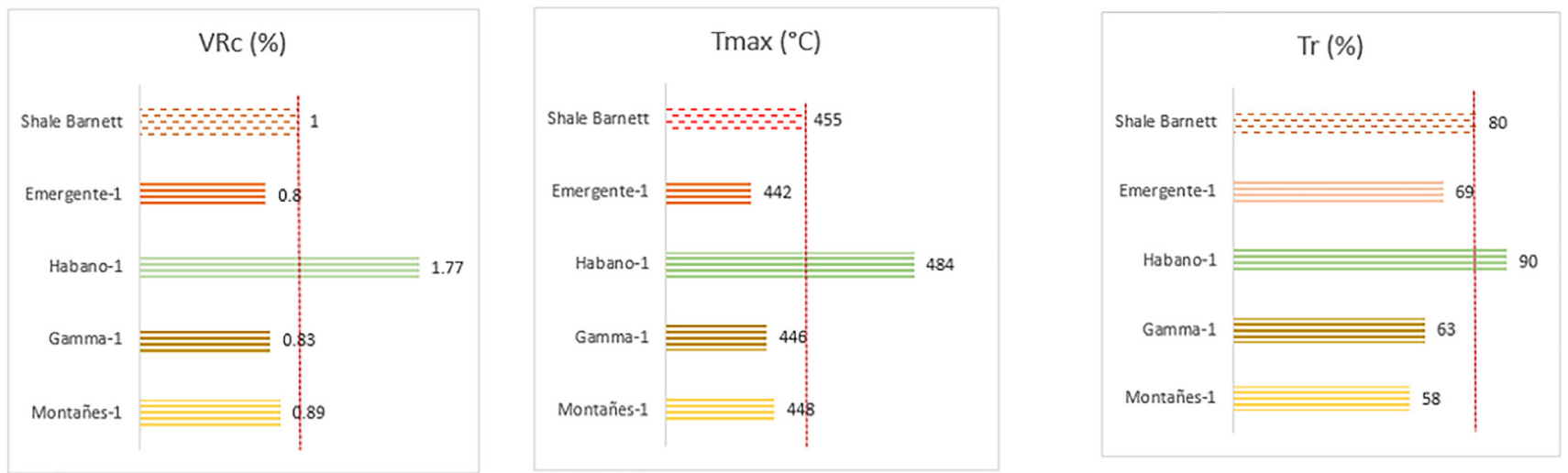

Figura 10 Gráficos de los valores Ro, Tr y Tmax de los pozos estudiados vs. los valores de corte utilizados en la cuenca de Barnett. 


\section{NOMENGLATURA}

$\mathrm{IH}$ Índice de Hidrógeno [mgHC/g COT]

IO Índice de Oxigeno [mgCO2/g COT]

PP Potencial Productor

VRo Reflectancia de la Vitrinita [\%]

S1 Hidrocarburos libres [mg HC/g roca]

S2 Kerogen Yield [mg HC/g roca]

Tmax Temperatura Máxima (pirólisis) $\left[{ }^{\circ} \mathrm{C}\right]$

COT Carbono Orgánico Total [peso \%]

Tr Índice de Transformación [\%]

$\mathrm{V} \quad$ Índice de Heterogeneidad

\section{Referencias}

Barceló Duarte, J. ,1983, Lower Cretaceous stratigraphy and depositional system in North westwern Coahuila, Mxico,USA, The University of Texas at Austin, Tesis Doctoral $157 \mathrm{p}$.

CNH, 2016, Exploración y extracción de aceite y gas en lutitas, México, reporte técnico. Comisión Nacional de Hidrocarburos.

DOE/EIA, 2016, Annual Energy Outlook 2016 with projections to 2040, USA, documento técnico, 438p. https://www.eia.gov/ outlooks/aeo/pdf/0383(2016).pdf

Humphrey, W. E., 1958, Estudio de la estratigrafía del Mesozoico y de la Tectónica de la Sierra Madre Oriental entre Monterey N.L. y Torreón Coah.; Estudio de la cuenca carbonifera de Sabinas, Coah. Visita a las Grutas de García. Morfología típica de Bolson y observación del tipo de pliegues en la Sierra de Parras: Boletín de la Asociación
Mexicana de Geólogos Petroleros 5(1-2), 5-102.

Jarvie, D.M., 2005, Assessment of the gas potential and yields from shales: The Barnett Shale model, in B. Cardott, ed., Oklahoma Geological Survey Circular 110: Unconventional Gas of the Southern Mid-Continent Symposium, March 9-10:, Oklahoma City, Oklahoma, p. 37-50.

Jarvie, D.M., 2007, Unconventional shale-gas systems: The Mississippian Barnett Shale of north-central Texas as one model for thermogenic shale-gas assessment: AAPG Bulletin, 91 (4), 475-499. https://doi. org/10.1306/12190606068

Maschio, G., 2003, A new upscaling technique based on Dykstra - Parsons coefficient: evaluation whith streamline reservoir simulation: Journal of petroleum Sciencie and Engineering 40 (1-2), 27 -36. https:// doi.org/10.1016/S0920-4105(03)00060-3

Meneses S. J., 2015, Lessons Learned in the Eagle Ford Play and Applicability to Mexico, USA, The University of Texas at Austin, Tesis Doctoral 182p.

Padilla y Sánchéz R. J., 1982, Geologic Evolution of the Sierra Madre Oriental, Between Linares, Concepción del Oro, Saltillo and Monterrey, Mexico, USA, The University of Texas at Austin, Tesis Doctoral 238p.

Tellez, A. M., 2011, Evaluación geológica geoquímica de las lutitas gasíferas del play Eagle Ford - Agua Nueva, en la plataforma de Tamaulipas y las cuencas de Sabinas y Burgos. Coordinación de Prospectos y Caractarización Inicial PEMEX, México. 